\title{
On the Mittag-Leffler Property
}

\author{
M. A. Alshumrani*
}

Department of Mathematics, King Abdulaziz University, P.O. Box 80203 Jeddah 21589, K.S.A

\begin{abstract}
Let $C$ be a category with strong monomorphic strong coimages, that is, every morphism $f$ of $C$ factors as $f=u \circ g$ so that $g$ is a strong epimorphism and $u$ is a strong monomorphism and this factorization is universal. We define the notion of strong Mittag-Leffler property in pro- $C$. We show that if $f: X \rightarrow Y$ is a level morphism in pro- $C$ such that $p(Y)_{\alpha}^{\beta}$ is a strong epimorphism for all $\beta>\alpha$, then $X$ has the strong Mittag-Leffler property provided $f$ is an isomorphism. Also, if $f: X \rightarrow Y$ is a strong epimorphism of pro- $C$ and $X$ has the strong Mittag-Leffler property, we show that $Y$ has the strong Mittag-Leffler property. Moreover, we show that this property is invariant of isomorphisms of pro- $C$.
\end{abstract}

Keywords: Pro-categories, strong Mittag-Leffler property, categories with strong monomorphic strong coimages. MSC: Primary 16B50.

\section{INTRODUCTION}

In [1], J. Dydak and F. R. Ruiz del Portal generalized the notion of Mittag-Leffler property to arbitrary balanced categories with epimorphic images. They obtained several results.

In [2], the author defined the notion of categories with strong monomorphic strong coimages. $C$ is a category with strong monomorphic strong coimages if every morphism $f$ of $C$ factors as $f=u \circ g$ so that $g$ is a strong epimorphism and $u$ is a strong monomorphism and this factorization is universal among such factorization. In this paper, we define the notion of strong Mittag-Leffler property in pro- $C$. We show that if $f: X \rightarrow Y$ is a level morphism in pro- $C$ such that $p(Y)_{\alpha}^{\beta}$ is a strong epimorphism for all $\beta>\alpha$, then $X$ has the strong Mittag-Leffler property provided $f$ is an isomorphism (Theorem 3.2). Also, if $f: X \rightarrow Y$ is a strong epimorphism of pro- $C$ and $X$ has the strong Mittag-Leffler property, we show that $Y$ has the strong Mittag-Leffler property (Corollary 3.6). Moreover, we show that this property is invariant of isomorphisms of pro- $C$ (Corollary 3.5).

\section{PRELIMINARIES}

First we recall some basic facts about pro-categories. The main reference is [3] and for more details see [4].

Let $C$ be an arbitrary category. Loosely speaking, the pro-category pro- $C$ of $C$ is the universal category with inverse limits containing $C$ as a full subcategory. An object of pro- $C$ is an inverse system in $C$, denoted by $X=\left(X_{\alpha}, p_{\alpha}^{\beta}, A\right)$, consisting of a directed set $A$, called the index

set, of $C$ objects $X_{\alpha}$ for each $\alpha \in A$, called the terms of $X$

*Address correspondence to this author at the Department of Mathematics, King Abdulaziz University, P.O. Box 80203, Jeddah 21589, K.S.A; Tel: (00966)26952297; E-mail: maalshmrani1@kau.edu.sa and of $C$ morphisms $p_{\alpha}^{\beta}: X_{\beta} \rightarrow X_{\alpha}$ for each related pair $\alpha<\beta$, called the bonding morphisms of $X$. A morphism of two objects $f: X=\left(X_{\alpha}, p_{\alpha}^{\beta}, A\right) \rightarrow Y=\left(Y_{\alpha^{\prime}}, p_{\alpha^{\prime}}^{\beta^{\prime}}, A^{\prime}\right)$ consists of a function $\varphi: A^{\prime} \rightarrow A$ and of morphisms $f_{\alpha^{\prime}}: X_{\varphi\left(\alpha^{\prime}\right)} \rightarrow Y_{\alpha^{\prime}}$ in $C$ one for each $\alpha^{\prime} \in A^{\prime}$ such that whenever $\alpha^{\prime}<\beta^{\prime}$, then there is $\quad \gamma \in A, \quad \gamma>\varphi\left(\alpha^{\prime}\right), \varphi\left(\beta^{\prime}\right) \quad$ for which $f_{\alpha^{\prime}} p_{\phi\left(\alpha^{\prime}\right)}^{\gamma}=p_{\alpha^{\prime}}^{\beta^{\prime}} f_{\beta^{\prime}} p_{\phi\left(\beta^{\prime}\right)}^{\gamma}$. From now onward, the index set $A$ of an object $X$ of pro- $C$ will be denoted by $I(X)$ and the bonding morphisms by $p(X)_{\alpha}^{\beta}$ for each $\alpha<\beta$.

If $P$ is an object of $C$ and $X$ is an object of pro- $C$, then a morphism $f: X \rightarrow P$ in pro- $C$ is the direct limit of Mor $\left(X_{\alpha}, P\right), \quad \alpha \in I(X)$ and so $f$ can be represented by $g: X_{\alpha} \rightarrow P$. Note that the morphism from $X$ to $X_{\alpha}$ represented by the identity $X_{\alpha} \rightarrow X_{\alpha}$ is called the projection morphism and denoted by $p(X)_{\alpha}$.

If $X$ and $Y$ are two objects in pro- $C$ with identical index sets, then a morphism $f: X \rightarrow Y$ is called a level morphism if for each $\alpha<\beta$, the following diagram commutes.

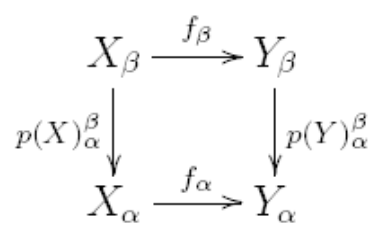

Theorem 2.1. For any morphism $f: X \rightarrow Y$ of pro- $C$ there exists a level morphism $f^{\prime}=X^{\prime} \rightarrow Y^{\prime}$ and isomorphisms $i: X \rightarrow X^{\prime}, j: Y^{\prime} \rightarrow Y$ such that $f=j \circ f^{\prime} \circ i$ and $I\left(X^{\prime}\right)$ is a 
cofinite directed set. Moreover, the bonding morphisms of $X^{\prime}$ (respectively, $Y^{\prime}$ ) are chosen from the set of bonding morphisms of $X$ (respectively, $Y$ ).

Recall that a morphism $f: X \rightarrow Y$ of $C$ is called a monomorphism if $f \circ g=f \circ h$ implies $g=h$ for any two morphisms $g, h: Z \rightarrow X$. A morphism $f: X \rightarrow Y$ of $C$ is called an epimorphism if $g \circ f=h \circ f$ implies $g=h$ for any two morphisms $g, h: Y \rightarrow Z$.

If $f$ is a morphism of $C$, then its domain will be denoted by $D(f)$ and its range will be denoted by $R(f)$. Hence, $f: D(f) \rightarrow R(f)$.

Next, we recall definitions of strong monomorphism and strong epimorphism and state some of their basic results obtained. The main reference is [3].

Definition 2.2. A morphism $f: X \rightarrow Y$ in pro- $C$ is called a strong monomorphism (strong epimorphism, respectively) if for every commutative diagram,

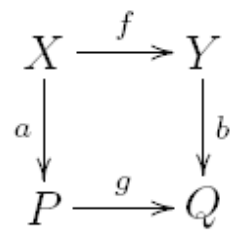

with $P, Q$ objects in $C$, there is a morphism $h: Y \rightarrow P$ such that $h \circ f=a(g \circ h=b$, respectively $)$.

Note that if $X$ and $Y$ are objects of $C$, then $f: X \rightarrow Y$ is a strong monomorphism (strong epimorphism, respectively) if and only if $f$ has a left inverse (a right inverse, respectively).

The following result presents the relation between monomorphisms and strong monomorphisms and between epimorphisms and strong epimorphisms.

Lemma 2.3. If $f$ is a strong monomorphism (strong epimorphism, respectively) of pro- $C$, then $f$ is a monomorphism (epimorphism, respectively) of pro- $C$.

Lemma 2.4. If $g \circ f$ is a strong monomorphism (strong epimorphism, respectively), then $f$ is a strong monomorphism ( $g$ is a strong epimorphism, respectively).

The following theorem is a characterization of isomorphisms in pro- $C$.

Theorem 2.5. Let $f: X \rightarrow Y$ be a morphism in pro- $C$. The following statements are equivalent.

(i) $\quad f$ is an isomorphism.

(ii) $\quad f$ is a strong monomorphism and an epimorphism.

Theorem 2.6. Suppose that,

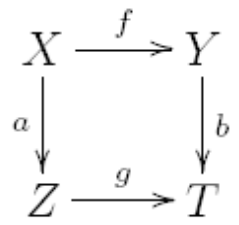

is a commutative diagram in pro- $C$. If $f$ is an epimorphism and $g$ is a strong monomorphism, then there is a unique morphism $h: Y \rightarrow Z$ such that $h \circ f^{\prime}=a$ and $g \circ h=b$.

In [2], the author defined the notion of categories with strong monomorphic strong coimages as follows.

Definition 2.7. $C$ is a category with strong coimages if every morphism $f$ of $C$ factors as $f=u \circ g$ so that $g$ is a strong epimorphism and this factorization is universal among such factorization, that is, given another factorization $f=v \circ h$ with $h$ being a strong epimorphism there is $t: D(v) \rightarrow D(u)$ such that $t \circ h=g$ and $u \circ t=v$.

Definition 2.8. $C$ is a category with strong monomorphic strong coimages if it is a category with strong coimages and $u$ in the universal factorization $f=u \circ g$ is a strong monomorphism.

We will need the following results which are proved in [2]. For this paper to be self-contained we include the results with their proofs.

Lemma 2.9. Let $C$ be any category. Then the following conditions on $C$ are equivalent:

(i) $\quad C$ is a category with strong monomorphic strong coimages.

Any morphism $f$ factors as $f=u \circ g$ so that $g$ is a strong epimorphism and $u$ is a strong monomorphism. Given another factorization $f=v \circ h$ with $h$ being a strong epimorphism and $v$ a strong monomorphism, there is an isomorphism $t: D(v) \rightarrow D(u)$ such that $t \circ h=g$ and $u \circ t=v$.

Proof. (i) $\Rightarrow$ (ii) Any morphism $f$ factors as $f=u \circ g$ such that $g$ is a strong epimorphism and $u$ is a strong monomorphism. Assume that $f$ have another factorization $f$ $=v \circ h$ with $h$ being a strong epimorphism and $v$ a strong monomorphism, there is $t: D(v) \rightarrow D(u)$ such that $t \circ h=g$ and $u \circ t=v$. Since $g$ is a strong epimorphism, $t$ is a strong epimorphism and hence an epimorphism by Lemma 2.3. Since $v$ is a strong monomorphism, $t$ is a strong monomorphism. Thus, $t$ is an isomorphism by Theorem 2.5.

(ii) $\Rightarrow$ (i) Any morphism $f$ factors as $f=u \circ g$ such that $g$ is a strong epimorphism and $u$ is a strong monomorphism. Now we show that $f$ is universal. Assume that $f=v \circ h$ is another factorization with $h$ a strong epimorphism. The morphism $v$ can be factored as $v=b \circ a$ where $a$ is a strong epimorphism and $b$ is a strong monomorphism, there is an isomorphism $c$ such that $c \circ a \circ h=g$ and $u \circ c=b$. Let $t=c \circ a$. Hence, the result holds.

By Lemma 2.9, any morphism $f$ of a category $C$ with strong monomorphic strong coimages has a unique, up to isomorphism, factorization into a composition $f=u \circ g$ where $g$ is a strong epimorphism and $u$ is a strong monomorphism. We write this unique factorization as $f=S M(f) \circ S E(f)$.

The range of $S E(f)$ will be called the image of $f$ and denoted by $1 \mathrm{~m}(f)$.

Theorem 2.10. Let $C$ be a category with strong monomorphic strong coimages. Let $f: X \rightarrow Y$ be a level 
morphism in pro- $C$. Then there exist level morphisms $g: X$ $\rightarrow Z$ and $h: Z \rightarrow Y$ such that $g_{\alpha}=\operatorname{SE}\left(f_{\alpha}\right), h_{\alpha}=S M\left(f_{\alpha}\right)$ for each $\alpha \in I(X)$ and $f=h \circ g$. Moreover, if $f$ is an isomorphism in pro- $C$, then both $h$ and $g$ are isomorphisms. Proof. First note that we have $f_{\alpha} \circ p(X)_{\alpha}^{\beta}=p(Y)_{\alpha}^{\beta} \circ f_{\beta}$ for $\beta>\alpha$. Since $C$ is a category with strong monomorphic strong coimages, we have,

$$
S M\left(f_{\alpha}\right) \circ S E\left(f_{\alpha}\right) \circ p(X)_{\alpha}^{\beta}=p(Y)_{\alpha}^{\beta} \circ S M\left(f_{\beta}\right) \circ S E\left(f_{\beta}\right) .
$$

This implies that the following diagram,

$$
\begin{gathered}
X_{\beta} \stackrel{S E\left(f_{\beta}\right)}{\longrightarrow} \operatorname{im}\left(f_{\beta}\right) \\
S E\left(f_{\alpha}\right) \circ p(X)_{\alpha}^{\beta} \downarrow \\
\operatorname{im}\left(f_{\alpha}\right) \stackrel{S M\left(f_{\alpha}\right)}{\longrightarrow} Y_{\alpha} p(Y)_{\alpha}^{\beta} \circ S M\left(f_{\beta}\right)
\end{gathered}
$$

is commutative in pro- $C$ with $S E\left(f_{\beta}\right)$ a strong epimorphism and $S M\left(f_{\alpha}\right)$ a strong monomorphism. Thus, there is a unique morphism $v: \operatorname{im}\left(f_{\beta}\right) \rightarrow \operatorname{im}\left(f_{\alpha}\right)$ by Theorem 2.6. Put $Z_{\alpha}=\operatorname{im}\left(f_{\alpha}\right)$ and $p(Z)_{\alpha}^{\beta}=v$. Thus, $Z$ is an object of pro- $C$. Further, put $g_{\alpha}=S E\left(f_{\alpha}\right)$ and $h_{\alpha}=S M\left(f_{\alpha}\right)$ for each $\alpha \in I(X)$. Hence, $f=h \circ g$. Obviously, $g$ is a strong epimorphism and $h$ is a strong monomorphism. Note that if $f$ is an isomorphism, hence a strong monomorphism by Theorem 2.5, then $g$ is a strong monomorphism by Lemma 2.4 and thus it is an isomorphism by Theorem 2.5. Also, if $f$ is an isomorphism, then $h$ is a strong epimorphism and thus it is an isomorphism. Hence, the result holds.

We denote $g$ by $S E(f)$ and $h$ by $S M(f)$. Therefore, we write $f$ as $S M(f) \circ S E(f)$.

\section{STRONG MITTAG-LEFFLER PROPERTY}

Definition 3.1. Let $C$ be a category with strong monomorphic strong coimages. An object $X$ of pro- $C$ has the strong Mittag-Leffler property if for every $\alpha \in I(X)$ there is $\beta>\alpha$ such that $S E\left(p(X)_{\alpha}^{\beta}\right) \circ p(X)_{\beta}^{\gamma}$ is a strong epimorphism for all $\gamma>\beta$.

$$
\text { We write } S E\left(p(X)_{\alpha}^{\beta}\right) \circ p(X)_{\beta}^{\gamma}=S E\left(p(X)_{\alpha}^{\gamma}\right) \text {. }
$$

Note that if $C$ is a category with strong monomorphic strong coimages and $X$ is an object of pro- $C$ such that $p(X)_{\alpha}^{\beta}$ is a strong epimorphism for all $\beta>\alpha$, then $X$ has the strong Mittag-leffler property. Indeed, $S E\left(p(X)_{\alpha}^{\beta}\right) \circ p(X)_{\beta}^{\gamma}$ is a strong epimorphism for all $\gamma>\beta$.
Theorem 3.2. Let $C$ be a category with strong monomorphic strong coimages. Let $f: X \rightarrow Y$ be a level morphism in pro- $C$ such that $p(Y)_{\alpha}^{\beta}$ is a strong epimorphism for all $\beta>\alpha$.

If $f$ is an isomorphism in pro- $C$, then $X$ has the strong Mittag-Leffler property.

Proof. Assume that $f$ is an isomorphism in pro- $C$. Then for each $\alpha$, there exists $\beta>\alpha$ and a morphism $r: Y_{\beta} \rightarrow X_{\alpha}$ in $C$ such that the following diagram commutes.

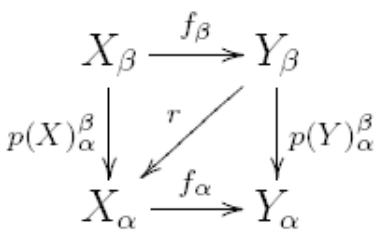

That is, $p(X)_{\alpha}^{\beta}=r \circ f_{\beta}$ and $p(Y)_{\alpha}^{\beta}=f_{\alpha} \circ r$. Since $p(Y)_{\alpha}^{\beta}$ is a strong epimorphism for all $\beta>\alpha$, we have $f_{\alpha}$ is a strong epimorphism by Lemma 2.4. For any $\gamma>\beta$, we have,

$$
r \circ p(Y)_{\beta}^{\gamma} \circ f_{\gamma}=r \circ f_{\beta} \circ p(X)_{\beta}^{\gamma}=p(X)_{\alpha}^{\beta} \circ p(X)_{\beta}^{\gamma}=p(X)_{\alpha}^{\gamma}
$$

Note that,

Therefore,

$$
r \circ p(Y)_{\beta}^{\gamma} \circ f_{\gamma}=S M(r) \circ S E(r) \circ p(Y)_{\beta}^{\gamma} \circ f_{\gamma}
$$

$$
\begin{aligned}
S E\left(p(X)_{\alpha}^{\gamma}\right)=S E(r) \circ p(Y)_{\beta}^{\gamma} \circ f_{\gamma} & =S E(r) \circ f_{\beta} \circ p(X)_{\beta}^{\gamma} \\
& =S E\left(p(X)_{\alpha}^{\beta}\right) \circ p(X)_{\beta}^{\gamma}
\end{aligned}
$$

Hence, $X$ has the strong Mittag-Leffler property.

We need the following special case of Theorem 2.10.

Lemma 3.3. Let $C$ be a category with strong monomorphic strong coimages. If $Y$ is an object of pro- $C$ and $e: I(Y) \rightarrow I(Y)$ is an increasing function, then there exist

level morphisms $g: X \rightarrow Z$ and $h: Z \rightarrow Y$ such that $g_{\alpha}=S E\left(p(Y)_{\alpha}^{e(\alpha)}\right)$ and $h_{\alpha}=S M\left(p(Y)_{\alpha}^{e(\alpha)}\right)$ for all $\alpha \in I(Y)$.

Moreover, both $g$ and $h$ are isomorphisms.

Proof. Let $Y$ be an object of pro- $C$ and $e: I(Y) \rightarrow I(Y)$ be an increasing function. Let $X_{\alpha}=Y_{e(\alpha)}$ and $p(X)_{\alpha}^{\beta}=p(Y)_{e(\alpha)}^{e(\beta)}$ for all $\alpha, \beta \in I(Y)$ with $\beta>\alpha$. Let $f: X \rightarrow Y$ be defined by $f_{\alpha}=p(Y)_{\alpha}^{e(\alpha)}$ for each $\alpha \in I(Y)$. Now if we continue as in the proof of Theorem 2.10, then the result holds.

Theorem 3.4. Let $C$ be a category with strong monomorphic strong coimages. If $Y$ has the strong Mittag-Leffler property and $I(Y)$ is cofinite, then there is a level morphism $h: Z \rightarrow Y$ in pro- $C$ such that $p(Z)_{\alpha}^{\beta}$ is a strong epimorphism for all $\beta>$ $\alpha$, each $h_{\alpha}$ is a strong monomorphism and $h$ is an isomorphism in pro- $C$. 
Proof. Suppose that $Y$ have the strong Mittag-Leffler property and $I(Y)$ is cofinite. Then for every $\alpha \in I(Y)$, there is $\beta>\alpha$ such that $S E\left(p(Y)_{\alpha}^{\beta}\right) \circ p(Y)_{\beta}^{\gamma}=S E\left(p(Y)_{\alpha}^{\gamma}\right)$ is a strong epimorphism for all $\gamma>\beta$. If we switch from $\beta$ to $\omega<\gamma$, then we have,

$$
\begin{aligned}
S E\left(p(Y)_{\alpha}^{\omega}\right) \circ p(Y)_{\omega}^{\gamma}=S E\left(p(Y)_{\alpha}^{\beta}\right) \circ p(Y)_{\beta}^{\omega} \circ p(Y)_{\omega}^{\gamma} & \\
& =S E\left(p(Y)_{\alpha}^{\beta}\right) \circ p(Y)_{\beta}^{\gamma}
\end{aligned}
$$

It is a strong epimorphism. But,

$$
\begin{aligned}
& S M\left(p(Y)_{\alpha}^{\omega}\right) \circ S E\left(p(Y)_{\alpha}^{\omega}\right) \circ p(Y)_{\omega}^{\gamma}=p(Y)_{\alpha}^{\omega} \circ p(Y)_{\omega}^{\gamma}=p(Y)_{\alpha}^{\gamma} \\
& \text { Thus, } \quad S E\left(p(Y)_{\alpha}^{\omega}\right) \circ p(Y)_{\omega}^{\gamma}=S E\left(p(Y)_{\alpha}^{\gamma}\right) \text { is a strong }
\end{aligned}
$$

epimorphism for $\gamma>\omega$. Therefore, by induction on the number of predecessors $n(\alpha)$ of $\alpha \in I(Y)$, we can construct an increasing function $e: I(Y) \rightarrow I(Y)$ such that $S E\left(p(Y)_{\alpha}^{e(\alpha)}\right) \circ p(Y)_{e(\alpha)}^{\gamma}=S E\left(p(Y)_{\alpha}^{\gamma}\right)$ is a strong epimorphism for all $\gamma>e(\alpha)$. Using Lemma 3.3, there exist level morphisms $g: X \rightarrow Z$ and $h: Z \rightarrow Y$ such that $g_{\alpha}=S E\left(p(Y)_{\alpha}^{e(\alpha)}\right)$ and $h_{\alpha}=S M\left(p(Y)_{\alpha}^{e(\alpha)}\right)$ for all $\alpha \in I(Y) . h$ is an isomorphism. Note that $p(Z)_{\alpha}^{\beta}$ is a strong epimorphism for all $\beta>\alpha$ since,

$p(Z)_{\alpha}^{\beta} \circ S E\left(p(Y)_{\beta}^{e(\beta)}\right)=S E\left(p(Y)_{\alpha}^{e(\alpha)}\right) \circ p(Y)_{e(\alpha)}^{e(\beta)} \quad$ is $\quad$ a strong epimorphism. Hence, the theorem is proved.

Corollary 3.5. Let $C$ be a category with strong monomorphic strong coimages. If $X$ is isomorphic to $Y$ in pro- $C$ and $Y$ has the strong Mittag-Leffler property, then $X$ has the strong Mittag-Leffler property.

Proof. Assume that $f: X \rightarrow Y$ is an isomorphism in pro- $C$ and $Y$ have the strong Mittag-Leffler property. Using Theorem 2.1, we can see that $Y^{\prime}$ has the strong Mittag-Leffler property if and only if $Y$ has the strong Mittag-Leffler property since the bonding morphisms of $Y^{\prime}$ are chosen from the bonding morphisms of $Y$. Therefore, we may assume that $p\left(Y^{\prime}\right)_{\alpha}^{\beta}$ is a strong epimorphism for all $\beta>\alpha$ by Theorem 3.4. There exists a level isomorphism $f^{\prime}: X^{\prime} \rightarrow Y^{\prime}$ by Theorem 2.1. Thus, $X^{\prime}$ has the strong Mittag-Leffler property by Theorem 3.2. Hence, $X$ has the strong Mittag-Leffler property.

Corollary 3.6. Let $C$ be a category with strong monomorphic strong coimages. If $f: X \rightarrow Y$ is a strong epimorphism of pro- $C$ and $X$ has the strong Mittag-Leffler property, then $Y$ has the strong Mittag-Leffler property.

Proof. Assume that $f: X \rightarrow Y$ is a strong epimorphism of pro- $C$ and $X$ have the strong Mittag-Leffler property. Using Theorem 3.4, we may assume that $f$ is a level morphism of pro- $C$ such that each $p(X)_{\alpha}^{\beta}$ is a strong epimorphism. Note that there exist level morphisms $S E(f): X \rightarrow Z$ and $S M(f): Z \rightarrow Y$ such that each $S E(f)_{\alpha}$ is a strong epimorphism and each $S M(f)_{\alpha}$ is a strong monomorphism and $f=S M(f) \circ S E(f)$ by Theorem 2.10. Since $S E(f)_{\alpha} \circ p(X)_{\alpha}^{\beta}=p(Z)_{\alpha}^{\beta} \circ S E(f)_{\beta}$ is a strong epimorphism, we have $p(Z)_{\alpha}^{\beta}$ is a strong epimorphism by Lemma 2.4. Since $f$ is a strong epimorphism, we have $S M(f)$ is a strong epimorphism and thus it is an isomorphism by Theorem 2.5. Hence, $Y$ has the strong Mitttag-Leffler property by Theorem 3.2 .

\section{ACKNOWLEDGEMENTS}

I thank King Abdulaziz University for supporting this work.

\section{REFERENCES}

[1] Dydak J, Ruiz del Portal FR. Monomorphisms and epimorphisms in pro-categories. Topol Appl 2007; 154: 2204-22.

[2] Alshumrani MA. Categories with strong monomorphic strong coimages. Missouri J Math Sci 2011; in press.

[3] Dydak J, Ruiz del Portal FR. Isomorphisms in pro-categories. J Pure Appl Algebra 2004; 190: 85-120.

[4] Marde $\tilde{S}$ ic S, Segal J. Shape Theory. North-Holland: Amsterdam 1982 .

This is an open access article licensed under the terms of the Creative Commons Attribution Non-Commercial License (http://creativecommons.org/licenses/by$\mathrm{nc} / 3.0 /$ ), which permits unrestricted, non-commercial use, distribution and reproduction in any medium, provided the work is properly cited. 